\title{
Transmembrane protein 108 involves in adult neurogenesis in the hippocampal dentate gyrus
}

\author{
Zheng Yu ${ }^{1,2 \dagger}$, Dong Lin ${ }^{1 \dagger}$, Yanzi Zhong ${ }^{1}$, Bin Luo ${ }^{1}$, Shengsheng Liư ${ }^{3}$, Erkang Fei ${ }^{1}$, Xinsheng Lai ${ }^{1}$, Suqi Zou ${ }^{1}$ \\ and Shunqi Wang ${ }^{1,2^{*}}$ (D)
}

\begin{abstract}
Background: Transmembrane protein 108 (Tmem108) is a risk gene of psychiatric diseases including schizophrenia, bipolar disorder and major depression disorder. However, the pathophysiological mechanisms of Tmem108 are largely unknown.

Results: Here we investigated the pathophysiological function of Tmem108 in the hippocampal dentate gyrus by using Tmem 108 mutant mice. Tmem108 highly expressed in the dentate gyrus and CA3 of the hippocampus. Dentate gyrus is a brain region where adult neurogenesis occurs, and aberrant adult neurogenesis in dentate gyrus has been implicated in major depression disorder. Indeed, Tmem 108 mutant mice had lower immobility than wild type mice in tail suspension test and forced swimming test. BrdU and anti-Ki67 antibody staining indicated that adult neurogenesis of the hippocampal dentate gyrus region decreased in Tmem 108 mutant mice. qPCR results showed that expression of Axin2, DISC1 and $\beta$-Catenin, three dentate gyrus adult neurogenesis related genes in Wnt/ $\beta$-Catenin signaling pathway, decreased in Tmem 108 mutant mice. Furthermore, Tmem108 enhanced free $\beta$-Catenin level in dual luciferase assay.
\end{abstract}

Conclusions: Thus, our data suggest that Tmem108 increases adult neurogenesis and plays a complexity role in psychiatric disorders.

Keywords: Transmembrane protein 108, Adult neurogenesis, Dentate gyrus, Major depression disorder, Bipolar disorder

\section{Introduction}

Adult neurogenesis, the generation of functional neurons from adult neural progenitors, mainly occurs in subventricular zone (SVZ) of the lateral ventricle and subgranular zone (SGZ) of the hippocampal dentate gyrus (DG) [1-3]. Many molecular pathways involve in adult neurogenesis [4], such as Shh, Notch and Wnt signaling pathway. Wnt/ $\beta$-Catenin signaling pathway has an important role in promoting proliferation of adult neuronal stem

\footnotetext{
*Correspondence: wsqi@ncu.edu.cn

†Zheng Yu and Dong Lin contributed equally to this work

${ }^{1}$ Laboratory of Synaptic Development and Plasticity, Institute of Life Science and School of Life Sciences, Nanchang University, Nanchang, Jiangxi, China

Full list of author information is available at the end of the article
}

cells (or precursor cells) and enhancing differentiation of neuronal precursor cells [4].

Aberrant adult neurogenesis in DG has been implicated in major depression disorder (MDD); in addition, enhancement of adult neurogenesis is regarded as one of the efficient indexes to MDD treatment [5, 6]. MDD, according to world health organization statistics in 2017, as a common mental disorder, affected more than 300 million people worldwide [7]. Severe MDD leads to suicide, and it gives society and family heavy burden and great harm [7].

Transmembrane protein 108 (Tmem108), also named Retrolinkin [8-10], locates on the human chromosome 3q21. Tmem108 expression is found as early as E8.5 in central nervous system $[11,12]$ and it plays an important 
role in central nervous system [8-10, 13]. Genome-wide association study (GWAS) found that TMEM108 is a susceptibility gene of psychiatric disorder, including schizophrenia, bipolar disorder (BPD) and MDD [14-17].

In the process of characterizing function of Tmem108 in psychiatric disorders, we found that Tmem108 is developmentally regulated and is required for glutamatergic transmission in DG [13]. We speculated that Tmem108 involved in MDD by regulating adult neurogenesis. In this study, the results showed that Tmem108 played a complexity role in psychiatric disorders, Tmem108 mutant mice had lower immobility than wild type mice in depression-like behavior tests, and adult neurogenesis of the hippocampal DG in Tmem108 mutant mice decreased. Further study indicated that Tmem108 could regulate adult neurogenesis by affecting Wnt/ $\beta$-Catenin signaling pathway.

\section{Materials and methods}

\section{Materials}

Tmem108 mutant (Tmem108-LacZ) mice were gifts from Dr. L. Mei, which were described previously [12, 13]. Mice were housed in a room 12-h light/dark cycle with ad libitum access to water and rodent chow diet.

The animal protocols in this study were approved by Nanchang University Medical Sciences Committee for research in vertebrate animals, in accordance with EN Directive 2010/63/EU on the protection of animals used for scientific purposes [18]. For in vivo experiment, surgery was performed under sodium pentobarbital anesthesia $(50 \mathrm{mg} / \mathrm{kg}$, ip injection), and all efforts were made to minimize suffering. After terminal experiments, mice were euthanized by carbon dioxide inhalation followed by cervical dislocation.

\section{Reagents}

X-gal (5-Bromo-4-chloro-3-indoly $\quad \beta$-Dgalactopyranoside) was purchased from Sigma-Aldrich (cat. \#: B4252, $30 \mathrm{mg} / \mathrm{ml}$ for staining). BrdU (5-Bromo2'-deoxyuridine) was purchase from Sigma-Aldrich (cat. \#: B5002, $5 \mathrm{mg} / \mathrm{ml}$ for ip injection).

Antibodies information was as follows: anti-BrdU (Rat, Accurate Chemical \& Scientific Corporation, cat.\#: OBT0030; 1:1000 for staining); anti-Ki67 (Mouse, BD Biosciences cat.\#: 550609; 1:1000 for staining); Alexa Fluor 488 Donkey anti-rat lgG (Thermo Fisher Scientific, cat.\#: A-21208; 1:1000 for staining), Alexa Fluor 568 goat anti-rabbit $\lg$ (Thermo Fisher Scientific, cat.\#: A11011; 1:1000 for staining); anti- $\beta$-Actin antibody (Rabbit, Santa Cruz Biotechnology cat.\#: sc-1616-R; 1:2000 for blotting); anti-Tmem108 antibody (Rabbit, 1:1000 for blotting) was kindly provided by Dr. J. Liu [8]. Polyclonal horseradish peroxidase (HRP)-conjugated goat anti-rabbit IgG (cat.
\#: 32260) and goat anti-mouse IgG (cat. \#: 32230) secondary antibodies were purchased from Pierce Thermo Fisher Scientific (1:2000 for blotting).

The construct pFlag-cmv1-Wnt3a was described, previously [18]. The construct pFlag-cmv1-Tmem108 was described previously [13]. The vector $\mathrm{p} 3 \times$ Flag-cmv- 24 was kept by our lab.

\section{Cell line and culture condition}

HEK293 cell line was kindly provided by Dr. S. Luo and the cells were maintained in Dulbecco's modified Eagle medium (DMEM; 4.5 g/l D-glucose, Thermo Fisher Scientific) supplemented with $10 \%$ fetal bovine serum (Thermo Fisher Scientific) and $1 \times$ Penicillin-Streptomycin Solution (Thermo Fisher Scientific) in tissue culture dishes in a humidified incubator at $37{ }^{\circ} \mathrm{C}$ with an atmosphere of $95 \%$ air and $5 \%$ carbon dioxide.

\section{In vitro transfection and dual luciferase reporter assay}

All transfection was performed using lipofectamine 3000 transfection reagent, according to the manufacturer's instructions (Thermo Fisher Scientific). Firefly luciferase reporter vectors (TOP-Flash and Fop-Flash) were generous gifts form Dr. L. Mei, as well as Renilla luciferase vector pRL-TK, which utilized as a transfection internal control.

HEK293 cells were seeded into 24-well plate. Total $760 \mathrm{ng}$ plasmids DNA/well were transfected, using ratio of $\mathrm{p} 3 \times$ Flag-cmv-24 or pFlag-cmv1-Wnt3a or pFlagcmv1-Tmem108: Firefly luciferase reporter vector (TOPFlash or Fop-Flash): pRL-TK=50: 25: 1. Each reaction was in triplicate wells.

\section{RNA extraction and qPCR}

Total RNA from mice tissues (wild mice $n=3$ ) and/or HEK293 cells was extracted using TRIzol Reagent (Invitrogen) according to the manufacturer's instructions. qPCR was performed as described previously [19]. The relative expression values were calculated relative to Gapdh or $\beta$-Actin by using the $2^{-\Delta C T}$ methods. Results were normalized to the control using the Microsoft Excel program. The qPCR primer sets were listed in Table 1.

\section{BrdU injection and BrdU staining}

BrdU injection (wild type mice $\mathrm{n}=5$, Tmem108 -/mice $\mathrm{n}=5$ ) and staining was performed as described previously [20] with minor modifications. In briefly, first, $\mathrm{BrdU}$ was dissolved in $0.9 \% \mathrm{NaCl}$ and ip injected three times at $1 \mathrm{~h}$ intervals at $100 \mathrm{mg} / \mathrm{kg}$ mice weight. Next, $24 \mathrm{~h}$ following the last injection, the brain was perfused and fixed with $4 \%$ paraformaldehyde (PFA). In order to denature DNA, frozen sections of the mice brain tissue were incubated in $1 \mathrm{M} \mathrm{HCL}$ for $30 \mathrm{~min}$ at room 
Table 1 Primer sequence used in qPCR assays

\begin{tabular}{|c|c|c|}
\hline Gene name & Forward primer sequence $\left(5^{\prime}->3^{\prime}\right)$ & Reverse primer sequence $\left(5^{\prime}->3^{\prime}\right)$ \\
\hline Tmem108 & CCTGAGCTACTGGAACAATGCC & CAGTGTCTCGATAGTCGCCATTG \\
\hline Gapdh & CATCACTGCCACCCAGAAGACTG & ATGCCAGTGAGCTTCCCGTTCAG \\
\hline$\beta$-Actin & CATTGCTGACAGGATGCAGAAGG & TGCTGGAAGGTGGACAGTGAGG \\
\hline Disc1 & TGGTCGAGGATGGCGATTACGA & AGAGCAGGTTGCTGTGAAGGCA \\
\hline$\beta$-Catenin & GTTCGCCTTCATTATGGACTGCC & ATAGCACCCTGTTCCCGCAAAG \\
\hline Apc & GTGGACTGTGAGATGTATGGGC & CACAAGTGCTCTCATGCAGCCT \\
\hline Gsk3 $\beta$ & GAGCCACTGATTACACGTCCAG & CCAACTGATCCACACCACTGTC \\
\hline PI3K & CAAACCACCCAAGCCCACTACT & CCATCAGCAGTGTCTCGGAGTT \\
\hline Axin1 & GTCCAGTGATGCTGACACGCTA & GCCCATTGACTTGGATACTCTCC \\
\hline Axin2 & ATGGAGTCCCTCCTTACCGCAT & GTTCCACAGGCGTCATCTCCTT \\
\hline
\end{tabular}

temperature, and acid was neutralized by rinsing section three times with PBST (0.1 M PBS (phosphate buffer saline) containing $0.1 \%$ Triton X-100). After blocked in blocking buffer (containing 5\% goat serum, 2\% BSA, $0.1 \%$ Triton $\mathrm{X}-100,0.1 \% \mathrm{NaN}_{3}$ in PBS, pH 7.4) for $1 \mathrm{~h}$ at room temperature, the section was incubated with BrdU primary antibody in antibody buffer (containing $2 \%$ goat serum, $1 \% \mathrm{BSA}, 0.1 \% \mathrm{NaN}_{3}$ in PBS, $\mathrm{pH} 7.4$ ) for $72 \mathrm{~h}$ at $4{ }^{\circ} \mathrm{C}$. And after rinsed three times with PBST, the sections were incubated with fluorochrome-conjugated secondary antibody in antibody buffer in the dark for $2 \mathrm{~h}$ at room temperature. Next, the sections were incubated with DAPI for $5 \mathrm{~min}$ and rinsed with PBS. At last, the sections were transferred to slides by a soft brush and were mounted with coverslips. The images were captured by inverted fluorescence microscope (Olympus FSX100).

\section{Immunohistochemistry (IHC)}

The mice brain (wild type mice $\mathrm{n}=4$, Tmem108 -/mice $n=4$ ) was perfused and fixed with $4 \%$ PFA, and the frozen sections were used for IHC similar to BrdU staining protocols as mentioned above with minor modification. Briefly, after washed three times with PBS, the fresh sections were blocked for $1 \mathrm{~h}$ at room temperature. Then, the sections were incubated with primary antibody overnight at $4{ }^{\circ} \mathrm{C}$. Next, the sections were rinsed three times with PBS, and incubated with secondary antibody in the dark for $2 \mathrm{~h}$ at room temperature. Finally, the sections were transferred to slides, and mounted with coverslips. The images were captured by inverted fluorescence microscope (Olympus FSX100).

\section{X-gal staining}

The mice (Tmem108 $+/-$ mice $\mathrm{n}=3$ ) brain was perfused and fixed with 4\% PFA, and the fresh frozen sections were used for X-gal staining. Firstly, the sections were washed with PBS containing $2 \mathrm{mM} \mathrm{MgCl}_{2}$ for $10 \mathrm{~min}$ at $4{ }^{\circ} \mathrm{C}$, and permeabilized with detergent rinse ( $2 \mathrm{mM} \mathrm{MgCl}, 0.01 \%$ sodium deoxycholate, $0.02 \% \mathrm{NP}-40$ in $0.1 \mathrm{M}$ phosphate buffer, pH 7.4) for $10 \mathrm{~min}$ at $4{ }^{\circ} \mathrm{C}$. Next, the sections were stained with staining solution $(5 \mathrm{mM}$ potassium ferrocyanide, $5 \mathrm{mM}$ potassium ferricyanide, $2 \mathrm{mg} / \mathrm{ml} \mathrm{X-gal} \mathrm{in}$ detergent rinse) overnight at $37{ }^{\circ} \mathrm{C}$. Then, after washed with PBS, the sections were added mount medium, and mounted with coverslips. Finally, the images were Z-stack taken by inverted fluorescence microscope (Olympus FSX100).

\section{Western blot}

Lysates of tissues were prepared in modified RIPA buffer ( $1 \times$ DPBS, $0.1 \%$ SDS, $0.5 \%$ sodium deoxycholate, $1 \%$ NP-40, $1 \mathrm{mM}$ PMSF, $1 \mathrm{mM}$ EDTA, $1 \mathrm{mg} / \mathrm{ml}$ aprotinin, leupeptin, and pepstatin A protease inhibitors). Samples were resolved by SDS-PAGE and transferred onto nitrocellulose membranes, which then were blocked with blocking buffer containing $5 \%$ nonfat dry milk in TBST buffer (20 mM Tris, $150 \mathrm{mM} \mathrm{NaCl}$ and $0.1 \%$ Tween 20) for $1 \mathrm{~h}$ at room temperature. Then, the membranes were washed with TBST buffer for 10 min three times before it was incubated with primary antibody overnight at $4{ }^{\circ} \mathrm{C}$. After washing three times with TBST, the membranes were incubated with HRP-coupled secondary antibody in blocking buffer for $1 \mathrm{~h}$ at room temperature. The immunoreacted bands were captured by enhanced chemiluminescence system (Bio RAD).

\section{Behaviors test}

In behavioral analysis, 10 - to 12 -week-old male mice were carried out by investigators unaware of mice genotype, and the mice were handled for habituation for 2 days before behavioral tests.

Open field test (wild type mice $\mathrm{n}=12$, Tmem108 -/mice $\mathrm{n}=10$ ) was performed as described previously $[13,21]$. In briefly, mice movements in the chamber 
$(50 \times 50 \times 80 \mathrm{~cm})$ were monitored by an overhead camera and tracking software (Video tracking interface version 1.85, Med Associate Inc.) for $30 \mathrm{~min}$. The mice used in open field test were reused one more time in forced swimming test (TST) or tail suspension test (FST).

For TST, mice (wild type mice $\mathrm{n}=12$, Tmem108 -/mice $\mathrm{n}=15$ ) were hung approximately $15 \mathrm{~cm}$ away from the bottom of the testing box for $6 \mathrm{~min}$. The immobility in the last 4 min was measured by sensory element controlled by computer software (Activity monitor version 6.02, Med Associate Inc.).

For FST, mice (wild type mice $\mathrm{n}=17$, Tmem108 -/mice $\mathrm{n}=10$ ) were forced to swim in a $2-1$ beaker filled with about $15 \mathrm{~cm}$ height water for $6 \mathrm{~min}$. Mice movement were monitored by horizontal camera and tracking software (Video freeze version 2.5.5.0, Med Associate Inc.). The immobility in the last 4 min was used for statistics.

\section{Statistical analysis}

Statistical analysis was conducted by GraphPad Prism 6.01. All data in each group were presented as mean with SEM (standard error of the mean) and data between groups were analyzed by two-tailed student $\mathrm{t}$-test. Difference was considered if the $\mathrm{p}$ value $<0.05$.

\section{Results}

\section{High Tmem 108 expression area in the brain}

We first evaluated the relative expression of Tmem108 in different tissues, RT-qPCR was used to check Tmem108 expression in adult wild type mice (Fig. 1A). As a classic internal control, Gapdh expression was used to normalize Tmem108 expression in seven selected tissues (heart, lung, liver, kidney, muscle, cerebrum cortex and cerebellum). Tmem108 expression in heart was defined as one, Tmem108 has a highly expression in liver, kidney, cerebrum cortex and cerebellum, comparing with the expression in heart and muscle (over 20 times).

LacZ staining was also used to examine Tmem108 expression in the brain. Tmem108 mutant mice targeting strategy was described before [13], in brief words, LacZ cassette including both stop code and a poly adenylation termination signal was inserted into the downstream of Tmem108 promoter (Fig. 1B). We found that Tmem108 had high expression in posteromedial cortical amygdaloid nucleus (PMCo), parafascicular thalamic nucleus (PTN), cortex, DG and CA3 of the hippocampus than other regions in brain coronal section slides (Fig. 1C).

Western blot confirmed Tmem108 expression between Tmem108 mutant mice and wild type mice (Fig. 1D). Nearly $90 \%$ of Tmem108 were successfully knockout in homozygous mutant mice (Tmem108 -/-), and Tmem108 expression in heterozygous mutant mice
(Tmem108 +/-) was consistent with theoretical estimation (50\% knockout).

\section{Decrease of immobility in Tmem 108 mutant mice}

As shown in Fig. 2, three kinds of animal behavior assays were carried out to investigate whether depression-like behaviors were altered in Tmem108 mutant mice, First, both total travel distance and duration in the center in open field test were not changed in Tmem108 mutant mice (Fig. 2a, b), which suggest that Tmem108 does not alter mice locomotor activity and anxious behavior. Then, both FST and TST were employed in depression-like tests (Fig. 2c, d), Tmem108 mutant mice exhibited lower immobility than wild type mice in both assays. These behavior tests suggest that depression-like behaviors in mice reduce in Tmem108 mutant mice.

\section{Decrease of DG adult neurogenesis in Tmem 108 mutant mice}

Figure 3 shows DG adult neurogenesis by BrdU staining assay and anti-Ki67 staining separately. Mice were injected ip with BrdU $24 \mathrm{~h}$ before sacrifice, and incorporated BrdU was detected in coronal sections by immunohistochemistry using an anti-BrdU antibody (Fig. 3a, c). BrdU positive cell numbers in the inner granule cell layer edge of the DG (per millimeter) and/or in DG granule cell layer (per square millimeter) decreased in Tmem108 mutant mice (Fig. 3b, d), which suggests that ablation of Tmem108 decreases DG neuronal progenitor cells proliferation.

Anti-Ki67 staining confirmed the result suggestion in BrdU assay (Fig. 3e, g). Without controversy, Ki67 positive cells in granule cell layer of the DG also decreased in Tmem 108 mutant mice (Fig. 3f, h). These indicate that Tmem108 increases adult neurogenesis in mice.

\section{Tmem 108 affects Wnt/ $\beta$-Catenin signaling pathway}

Lastly, expression profiling of $\mathrm{Wnt} / \beta$-Catenin signaling pathway were evaluated by RT-qPCR. On the premise of Gapdh expression as reference control, Axin2, Disc1 (Disrupted-in-Schizophrenia 1) and $\beta$-Catenin decreased in Tmem108 mutant mice hippocampus (Fig. 4a), which suggests that $\mathrm{Wnt} / \beta$-Catenin signaling pathway is disturbed in the hippocampus of Tmem108 mutant mice.

To further determine whether Tmem 108 involves classic Wnt/ $\beta$-Catenin signaling pathway, dual luciferase assay was carried out in vitro. In HEK293 cell culture, Tmem108 increased free $\beta$-Catenin level in the assay, though the activation was lower than the positive control Wnt3a (Fig. 4b), which suggests that Tmem108 enhances $W n t / \beta$-Catenin signaling pathway.

These data suggest that Tmem108 does not only increase adult neurogenesis in the hippocampal DG, but 
A

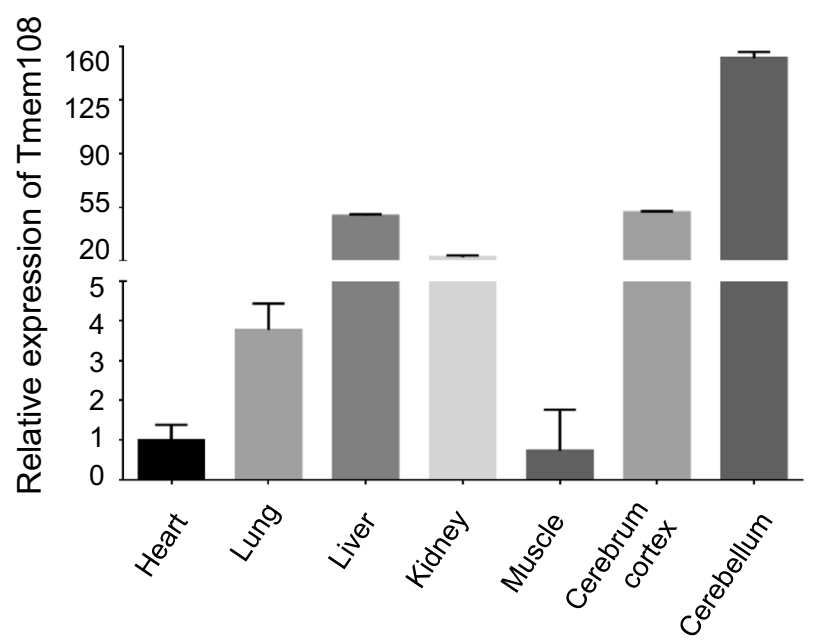

B

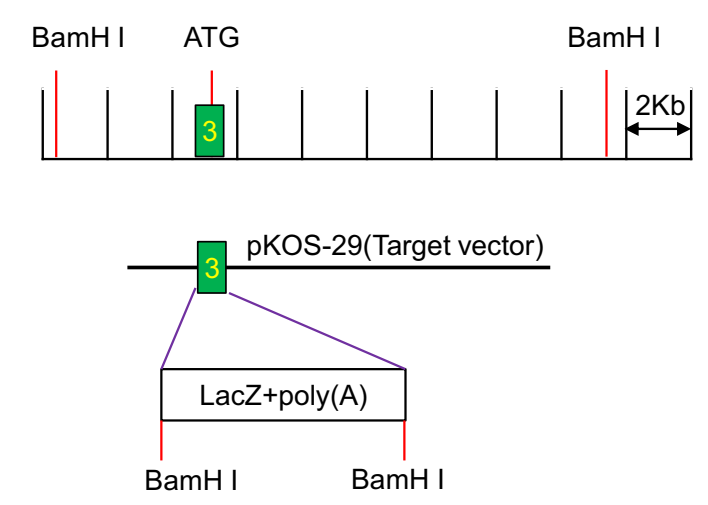

C
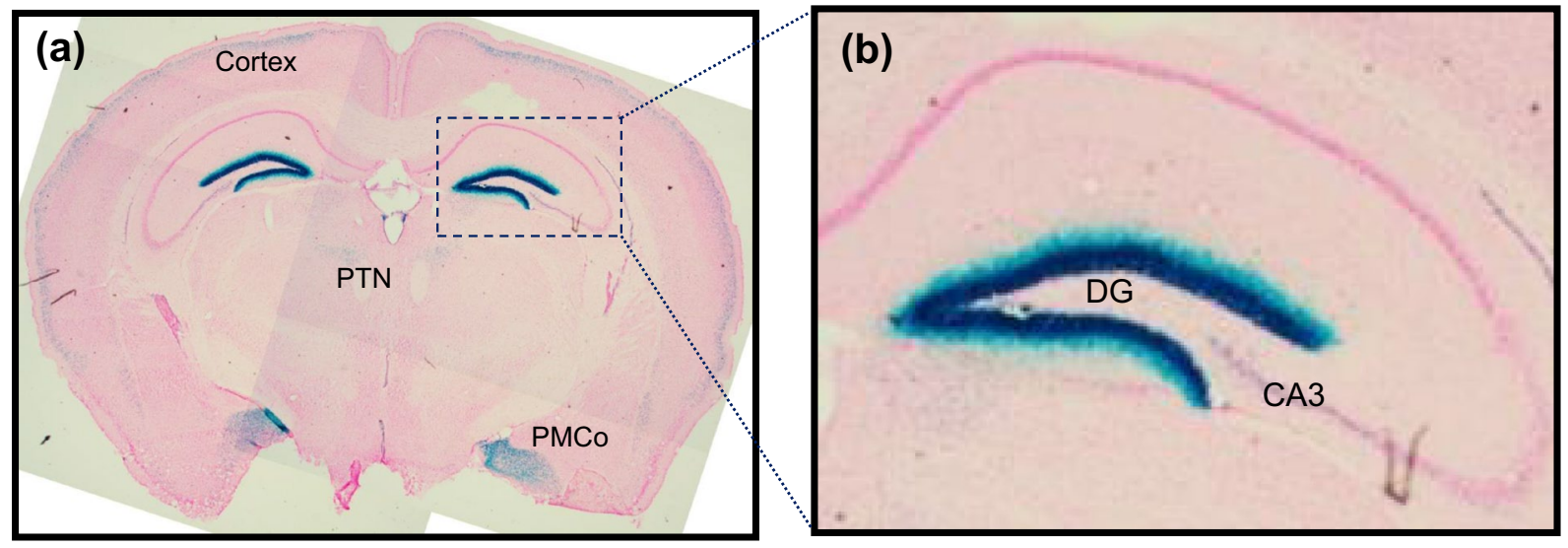

D

(a)

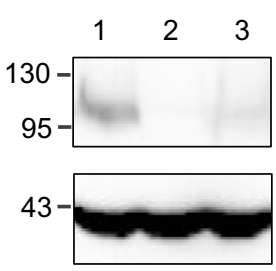

Short Exposure

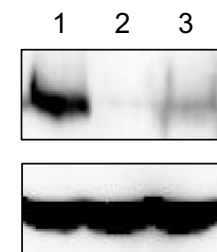

Anti-Tmem108

Anti- $\beta$-Actin (b)

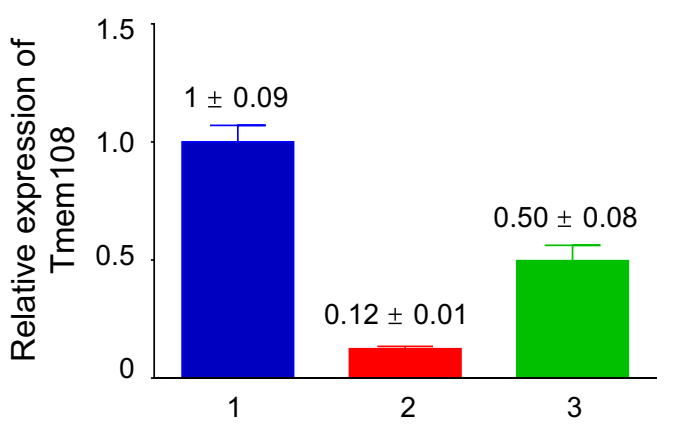

Fig. 1 High Tmem 108 expression area in mice brain. A Tmem 108 relative expression in different tissues in adult wild type mice (Gapdh expression as internal control, Tmem 108 expression in heart was defined as one, wild type mice $n=3$ ). B Tmem 108 mutant mice targeting strategy; the initiation code of Tmem 108 localizes in the exon 3, and LacZ gene is inserted into the downstream of Tmem108 promotor. C LacZ staining of Tmem 108 mutant mouse (Tmem108 +/-) hippocampus; PMCo: posteromedial cortical amygdaloid nucleus, PTN: Parafascicular thalamic nucleus. D Tmem 108 levels in hippocampus were evaluated by Western blotting assay; D (a) Representative image of Western blotting assays, $\mathbf{D}(b)$ Statistics of Tmem108 expression from Western blotting assay; 1 : wild type mice $n=3 ; 2: \operatorname{Tmem} 108$ knockout mice homozygous $(\operatorname{Tmem} 108-/-) n=3 ; 3$ : Tmem108 knockout mice heterozygous (Tmem108 +/-) n=3 
a

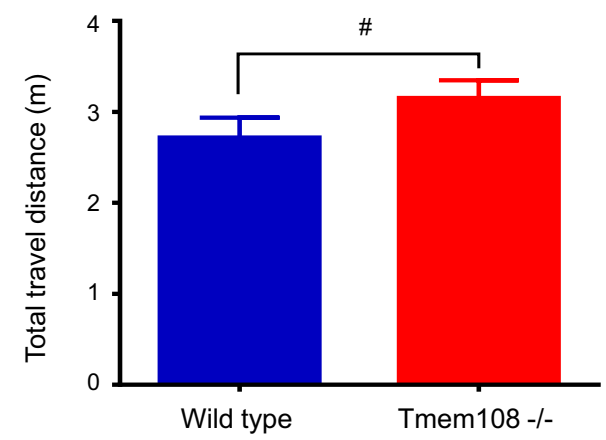

C

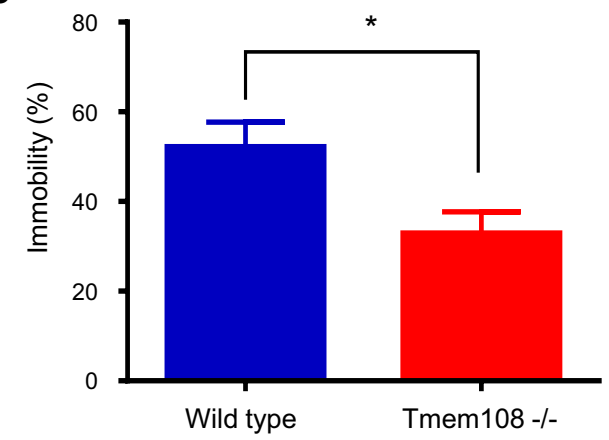

b

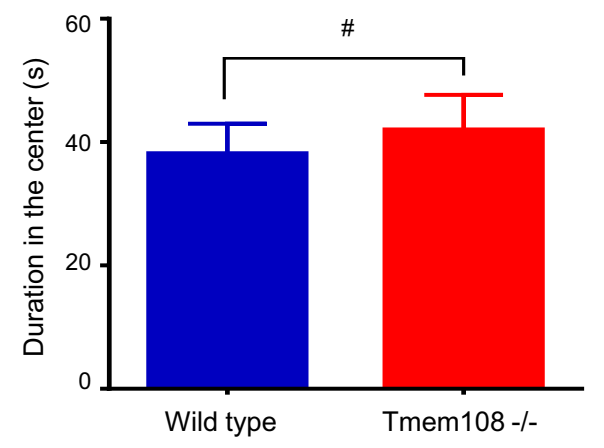

d

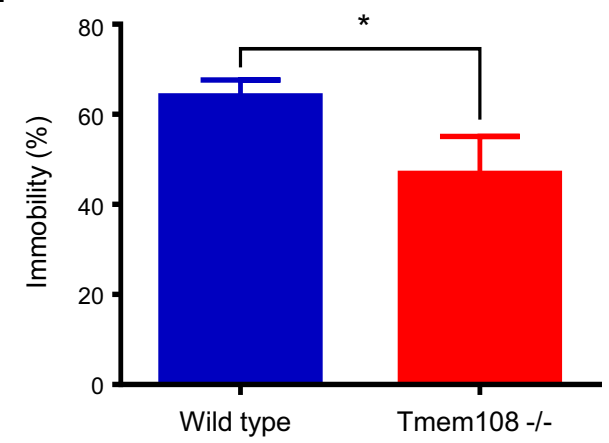

Fig. 2 Tmem 108 mutant mice had lower immobility. a Tmem 108 mutant mice locomotor activity was not different from wild type mice in open field test (total travel distance was no difference). b Tmem 108 mutant mice did not have anxious behavior in open field test (duration in the center was no difference, wild type mice, $n=12$, Tmem108 -/- mice, $n=10$ ). c Tmem 108 mutant mice had lower immobility in tail suspension test (TST, wild type mice $n=12$, Tmem $108-/-$ mice $n=15$ ). d Tmem 108 mutant mice had lower immobility in forced swimming test (FST, wild type mice $\mathrm{n}=17$, Tmem $108-/-$ mice $n=10)\left({ }^{*} p>0.05,{ }^{*} p<0.05\right)$

also affects Wnt/ $\beta$-Catenin signaling pathway. And furthermore, Tmem108 mutant mice behaviors in depression-like tests indicate that Tmem108 plays a complexity role in psychiatric disorders.

\section{Discussion}

In this study, the results indicate that Tmem108 highly expresses in DG and CA3 region of the hippocampus, and it acts as a positive signal in adult neurogenesis. Adult neurogenesis mainly occurs in SVZ and SGZ of DG region [1-3]. Because of convenient detection and easy quantification, DG region was frequently used to evaluate adult neurogenesis [5, 22, 23]. One of the hypotheses on MDD pathogenesis is decrease of adult neurogenesis [5, 23]. Accordingly, MDD usually exhibits deficiency on adult neurogenesis of DG region. Because adult neurogenesis decreases in Tmem108 mutant mice, Tmem108 mutant mice may be linked to depression.

Tmem108 mutant mice exhibit low immobility in both forced swimming test and tail suspension test, which look like anti-depression-like or mania-like behaviors. Therefore, adult neurogenesis decrease and anti-depression behavior are not consistent in Tmem108 mutant mice. The prevalent perspectives in

\footnotetext{
(See figure on next page.)

Fig. 3 Loss of Tmem108 decreased DG adult neurogenesis. Representative images of BrdU assay, the low panel was from the rectangular area of the upper panel in a (wild type mice $n=5$ ) or c (Tmem $108-/-$ mice $\mathrm{n}=5$ ); 2 -month mice were injected ip with $300 \mathrm{mg} / \mathrm{kg}$ BrdU $24 \mathrm{~h}$ before sacrifice; incorporated BrdU was detected in sections by IHC using an anti-BrdU antibody (green), nuclei were counterstained with DAPI (blue). Quantification of BrdU positive cell number per mm DG inner edge (b) and per square mm DG region (d). Representative images of anti-Ki67 staining (red) from 2-month mice, the low panel was from the rectangular area of upper panel in $\mathbf{e}$ (wild type mice $n=4)$ or $\mathbf{g}($ Tmem $108-/-$ mice $\mathrm{n}=4$ ); nuclei were counterstained with DAPI (blue). Quantification of Ki67 positive cell number per mm DG inner edge (f) and per square mm DG region $\left.(\mathbf{h}){ }^{* *} p<0.01,{ }^{* * *} p<0.001\right)$
} 
a
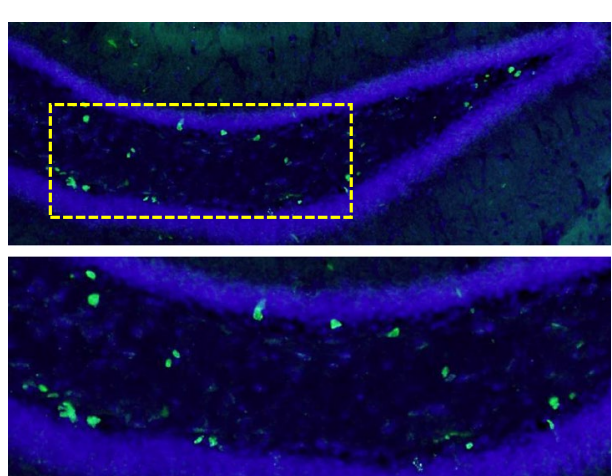

C
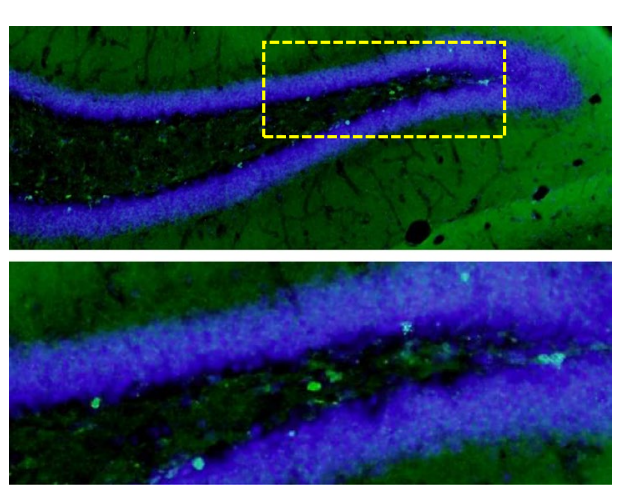

e
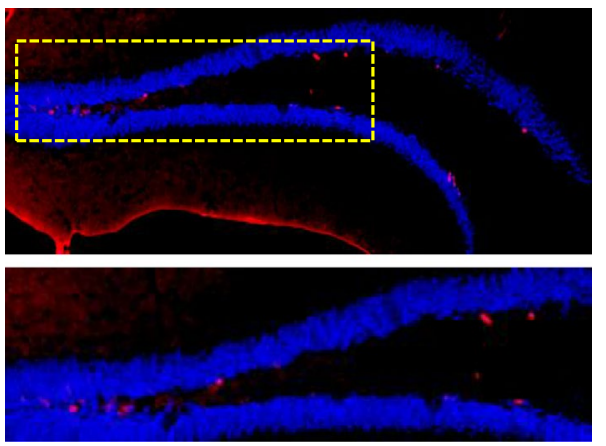

g

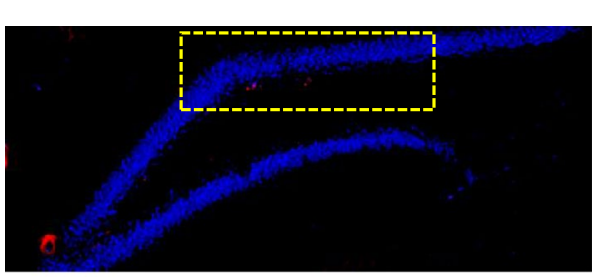

b

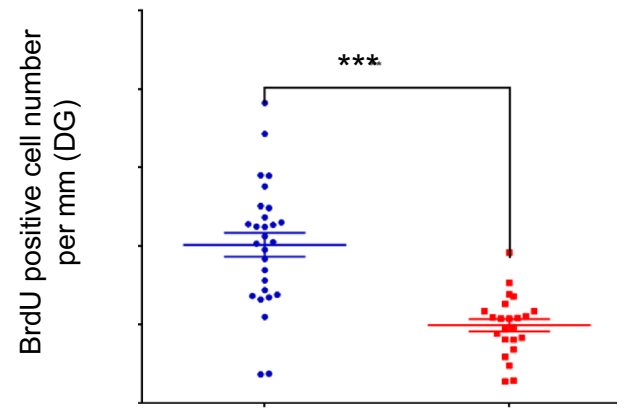

d

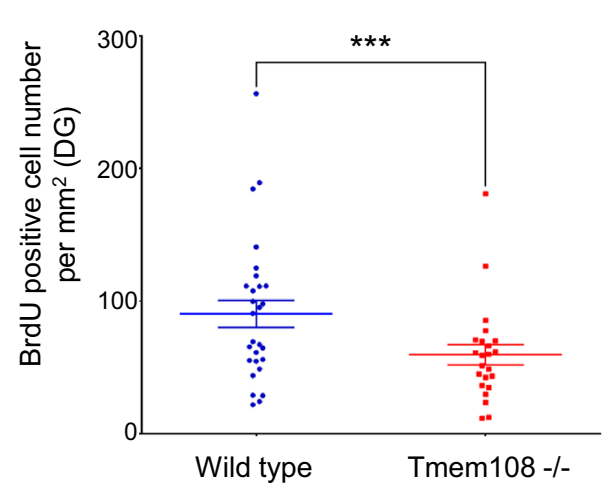

f

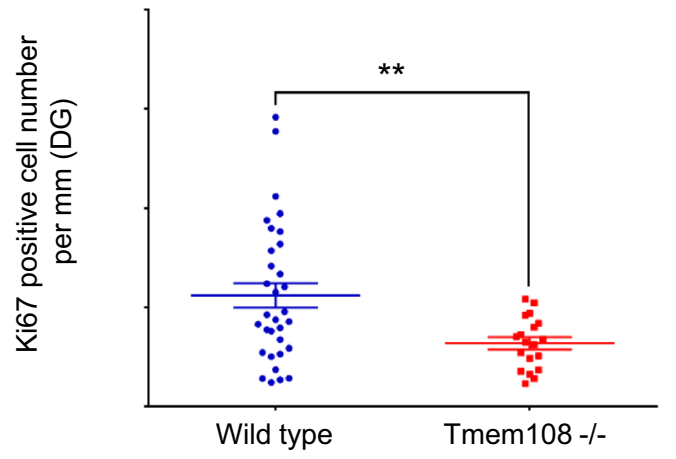

h

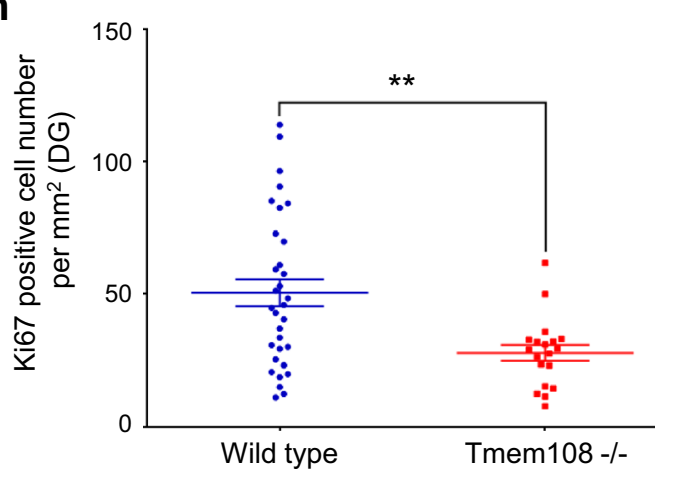


a

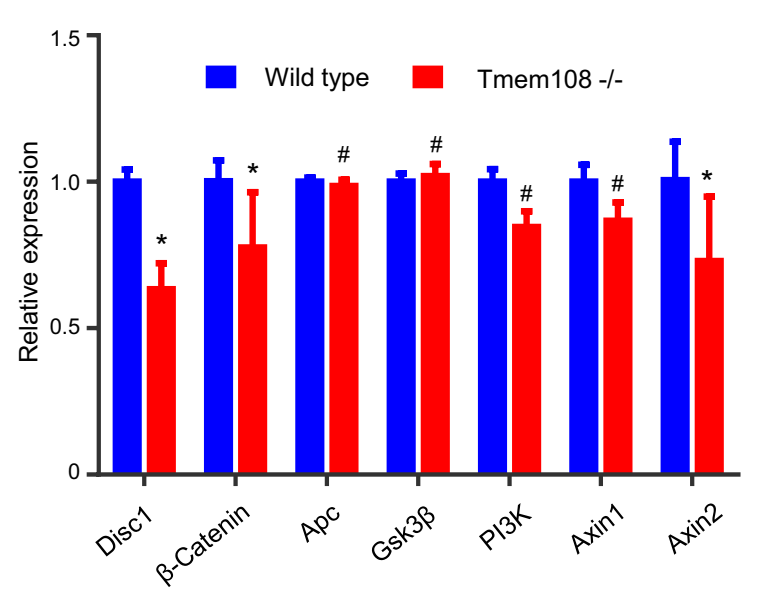

b

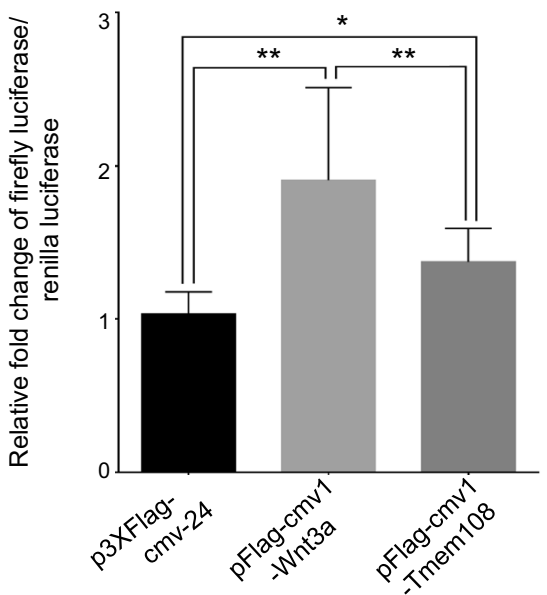

Fig. 4 Tmem 108 affected Wnt/ $\beta$-Catenin signaling pathway. a Expression profiling of Wnt signaling pathway by qPCR. Wnt signaling pathway was disturbed in hippocampus of Tmem 108 mutant mice; Gapdh mRNA expression was used as an internal control, mRNA expression relative to Gapdh in wild type was defined as one (wild type mice, $n=5$, Tmem $108-/-$ mice, $n=4,{ }^{*} p>0.05$, ${ }^{*} p<0.05$ ). b In HEK293 cell culture, Tmem108 increased free $\beta$-Catenin level in Dual luciferase assay. Relative TOP-Flash Firefly Luciferase (TFL1)/Renilla Luciferase (RL1) = Raw TFL1/Raw RL1; Relative FOP-Flash Firefly Luciferase (FFL2)/Renilla Luciferase (RL2)= Raw FFL2/Raw RL2; and final relative Firefly Luciferase (FL)/Renilla Luciferase (RL)= (Raw TFL1/Raw RL1)/(Raw FFL2/Raw RL2). Fold changes of FL/RL at p3 $\times$ Flag-cmv-24-treated condition is normalized as 1, and fold changes of FL/RL at pFlag-cmv1-Wnt3a-treated or pFlag-cmv1-Tmem108-treated condition is normalized to FL/RL at p3 $\times$ Flag-cmv-24-treated condition

MDD research hold that immobility is interpreted as a negative mood behavior $[5,24]$, and others do not agree with the opinion $[25,26]$. Tmem 108 mutant mice may habituate quickly, or have weak persistence in stress condition, and consequently, Tmem108 mutant mice show low immobility in stress environment.

In GWAS analysis, Tmem108 is related with BPD, MDD and schizophrenia [14-16], which share many similar behaviors. Low immobility may be a reflection of schizophrenia or BPD. It has been found that Tmem108 mutant mice have deficiency in prepulse inhibition test [13], which is a symbol of schizophrenia. Furthermore, in GWAS analysis, the $\mathrm{p}$ value in BPD and schizophrenia GWAS analysis are 0.00328 and 0.0024 , separately $[15,16]$, much higher than $\mathrm{p}$ value (0.0000148) in MDD [14]. BPD, formerly called mania depression, one of the hallmarks is the alternation of depression period and mania period [27]. Therefore, Tmem108 may play a complexity role in psychiatric disorders.

Low immobility in Tmem108 mutant mice may be an abnormal physiological manifestation induced by stress condition. To confirm the hypothesis, more behavior tests should be compared in normal condition with in stress condition. Whatever the cause of decrease of Tmem108 mutant mice immobility, it is unquestionable that adult neurogenesis in Tmem108 mutant mice decrease.
We wonder whether Tmem108 regulates adult neurogenesis by affecting Wnt// $\beta$-Catenin signaling pathway. Wnt/ $\beta$-Catenin signaling is a canonical pathway in control cell proliferation and differentiation, and it also involves adult neurogenesis [28-30]. In fact, several genes expression of Wnt/ $\beta$-Catenin signaling pathway decreased in Tmem108 mutant mice, such as $\beta$-Catenin, Axin2 and Disc1. Furthermore, in vitro dual luciferase assay suggests that Tmem108 increases free $\beta$-Catenin level. Free $\beta$-Catenin, as an intracellular signal transducer, is the main downstream executor of Wnt/ $\beta$-Catenin signaling pathway [31].

In conclusion, our study demonstrated that Tmem 108 involves in adult neurogenesis and it may regulate Wnt/ $\beta$-Catenin signaling pathway to affect adult neurogenesis. Furthermore, abnormal behaviors in Tmem108 mutant mice indicated that Tmem108 plays a complexity role in psychiatric disorders.

\section{Abbreviations}

BPD: bipolar disorder; DG: dentate gyrus; FST: forced swimming test; GWAS: genome-wide association study; IHC: immunohistochemistry; MDD: major depression disorder; PFA: paraformaldehyde; SGZ: subgranular zone; SVZ: subventricular zone; Tmem108: transmembrane protein 108; TST: tail suspension test; X-gal: 5-bromo-4-chloro-3-indoly $\beta$-D-galactopyranoside.

\section{Authors' contributions}

$Z Y$ and DL performed the experiments; SW conceived and designed the research; $Y Z, B L$ and $S L$ contributed to make the table and figures; $E F, X L$ and 
SZ contributed to the statistical analysis; SW wrote and revised the paper. All authors read and approved the final manuscript.

\section{Author details}

${ }^{1}$ Laboratory of Synaptic Development and Plasticity, Institute of Life Science and School of Life Sciences, Nanchang University, Nanchang, Jiangxi, China. ${ }^{2}$ School of Basic Medical Science, Nanchang University, Nanchang, Jiangxi,

China. ${ }^{3}$ Queen Mary School, Nanchang University, Nanchang, Jiangxi, China.

\section{Acknowledgements}

We thank Dr. Lin Mei (Case Western Reserve University) for selfless help in project development, members of the Lin Mei's lab for helpful discussions. We also thank Dr. Jiajia Liu (Institute of Genetics and Developmental Biology, Chinese Academy of Sciences) for providing anti-Tmem 108 antibody, and Dr. Shiwen Luo (The First Affiliated Hospital, Nanchang University) for providing HEK293 cell line.

\section{Competing interests}

The authors declare that they have no competing interests.

\section{Consent for publication}

Not applicable.

\section{Data availability statement}

Please contact the corresponding author for data on reasonable request.

\section{Ethics approval and consent to participate}

The animal protocols in this study were approved by Nanchang University Medical Sciences Committee (China) for research in vertebrate animal, in accordance with EN Directive 2010/63/EU on the protection of animals used for scientific purposes.

\section{Funding}

This work is supported in part by grants from National Natural Science Foundation of China (Grant Nos. 31460260, 31400936), and Scientific Research Foundation for Returned Scholars, Ministry of Education of China (To SW).

\section{Publisher's Note}

Springer Nature remains neutral with regard to jurisdictional claims in published maps and institutional affiliations.

Received: 2 January 2019 Accepted: 7 January 2019 Published online: 11 January 2019

\section{References}

1. Kempermann G, Song H, Gage FH. Neurogenesis in the adult hippocampus. Cold Spring Harbor Perspect Biol. 2015;7(9):a018812.

2. Aimone JB, Li Y, Lee SW, Clemenson GD, Deng W, Gage FH. Regulation and function of adult neurogenesis: from genes to cognition. Physiol Rev. 2014:94(4):991-1026.

3. Rusznak Z, Henskens W, Schofield E, Kim WS, Fu Y. Adult neurogenesis and gliogenesis: possible mechanisms for neurorestoration. Exp Neurobiol. 2016;25(3):103-12.

4. Liu H, Song N. Molecular mechanism of adult neurogenesis and its association with human brain diseases. J Cent Nerv Syst Dis. 2016;8:5-11.

5. Danzer SC. Depression, stress, epilepsy and adult neurogenesis. Exp Neurol. 2012;233(1):22-32.

6. Galecki P, Talarowska M, Anderson G, Berk M, Maes M. Mechanisms underlying neurocognitive dysfunctions in recurrent major depression. Med Sci Monit. 2015;21:1535-47.

7. World Health Organization. Depression from http://www.who.int/media centre/factsheets/fs369/en/. 2017.
8. Xu C, Fu X, Zhu S, Liu JJ. Retrolinkin recruits the WAVE1 protein complex to facilitate BDNF-induced TrkB endocytosis and dendrite outgrowth. Mol Biol Cell. 2016;27(21):3342-56.

9. Fu X, Yang Y, Xu C, et al. Retrolinkin cooperates with endophilin A1 to mediate BDNF-TrkB early endocytic trafficking and signaling from early endosomes. Mol Biol Cell. 2011;22(19):3684-98.

10. Liu JJ, Ding J, Wu C, et al. Retrolinkin, a membrane protein, plays an important role in retrograde axonal transport. Proc Natl Acad Sci USA. 2007:104(7):2223-8.

11. Miller RA, Christoforou N, Pevsner J, McCallion AS, Gearhart JD. Efficient array-based identification of novel cardiac genes through differentiation of mouse ESCS. PLoS ONE. 2008;3(5):e2176.

12. Tang T, Li L, Tang J, et al. A mouse knockout library for secreted and transmembrane proteins. Nat Biotechnol. 2010;28(7):749-55.

13. Jiao HF, Sun XD, Bates $R$, et al. Transmembrane protein 108 is required for glutamatergic transmission in dentate gyrus. Proc Natl Acad Sci USA. 2017;114(5):1177-82.

14. Major Depressive Disorder Working Group of the Psychiatric GC, Ripke S, Wray NR, et al. A mega-analysis of genome-wide association studies for major depressive disorder. Mol Psychiatry. 2013;18(4):497-511.

15. Cross-Disorder Group of the Psychiatric Genomics C. Identification of risk loci with shared effects on five major psychiatric disorders: a genomewide analysis. Lancet. 2013;381(9875):1371-9.

16. Schizophrenia Psychiatric Genome-Wide Association Study C. Genomewide association study identifies five new schizophrenia loci. Nat Genet. 2011:43(10):969-76.

17. Neale BM, Medland SE, Ripke S, et al. Meta-analysis of genome-wide association studies of attention-deficit/hyperactivity disorder. J Am Acad Child Adolesc Psychiatry. 2010:49(9):884-97.

18. Zong Y, Zhang B, Gu S, et al. Structural basis of agrin-LRP4-MuSK signaling. Genes Dev. 2012;26(3):247-58.

19. Wang $S$, Huang $S$, Zhao $X$, et al. Enrichment of prostate cancer stem cells from primary prostate cancer cultures of biopsy samples. Int J Clin Exp Pathol. 2014;7(1):184-93.

20. Wojtowicz JM, Kee N. BrdU assay for neurogenesis in rodents. Nat Protoc. 2006;1(3):1399-405

21. Wen L, LuYS, Zhu XH, et al. Neuregulin 1 regulates pyramidal neuron activity via ErbB4 in parvalbumin-positive interneurons. Proc Natl Acad Sci USA. 2010;107(3):1211-6.

22. Goncalves JT, Schafer ST, Gage FH. Adult neurogenesis in the hippocampus: from stem cells to behavior. Cell. 2016;167(4):897-914.

23. Christian KM, Song H, Ming GL. Functions and dysfunctions of adult hippocampal neurogenesis. Annu Rev Neurosci. 2014;37:243-62.

24. Hill AS, Sahay A, Hen R. Increasing adult hippocampal neurogenesis is sufficient to reduce anxiety and depression-like behaviors. Neuropsychopharmacology. 2015;40(10):2368-78.

25. Tanti A, Belzung C. Hippocampal neurogenesis: a biomarker for depression or antidepressant effects? Methodological considerations and perspectives for future research. Cell Tissue Res. 2013;354(1):203-19.

26. Lee MM, Reif A, Schmitt AG. Major depression: a role for hippocampal neurogenesis? Curr Top Behav Neurosci. 2013;14:153-79.

27. Niitsu T, Fabbri C, Serretti A. Predictors of switch from depression to mania in bipolar disorder. J Psychiatr Res. 2015:66-67:45-53.

28. Hussaini SM, Choi $\mathrm{Cl}$, Cho $\mathrm{CH}$, Kim HJ, Jun H, Jang MH. Wnt signaling in neuropsychiatric disorders: ties with adult hippocampal neurogenesis and behavior. Neurosci Biobehav Rev. 2014;47:369-83.

29. Varela-Nallar L, Inestrosa NC. Wnt signaling in the regulation of adult hippocampal neurogenesis. Front Cell Neurosci. 2013;7:100.

30. Zhang L, Yang X, Yang S, Zhang J. The Wnt/beta-catenin signaling pathway in the adult neurogenesis. Eur J Neurosci. 2011;33(1):1-8.

31. Pecina-Slaus N. Wnt signal transduction pathway and apoptosis: a review. Cancer Cell Int 2010:10:22. 